\title{
Uso y manejo de plaguicidas en diferentes sistemas de producción de fresa en México
}

\author{
Jesús Antonio Salazar Magallón ${ }^{1}$ - Carlos Eduardo Somoza Vargas ${ }^{2}$ - Beatriz Pérez Armendáriz \\ Mauricio Velásquez Soriano ${ }^{4}$ - Gerardo Torres García ${ }^{5}$ - Arturo Huerta de la Peña ${ }^{6}$ \\ Luis Daniel Ortega Martínez $7^{7}$
}

Recepción: 24/05/2017

Aceptación: 16/08/2017

\section{Resumen}

Se evaluó el uso y manejo de plaguicidas de distintos sistemas de producción en los Estados de Puebla y Tlaxcala, México. La metodología utilizada fue descriptiva, cualitativa y cuantitativa. Se entrevistó a informantes clave, los cuales representaron una superficie de cultivo promedio de $2000 \mathrm{~m}^{2}$. Se caracterizaron cinco sistemas de producción diferenciados por las tecnologías empleadas como riego localizado, sistema hidropónico y suelo con acolchado plástico, así como por el cultivo bajo condiciones de invernadero y libre exposición.

Los resultados muestran que el $100 \%$ de los productores y jornaleros utilizan plaguicidas; los empleados con mayor frecuencia son clasificados como poco probable de presentar riesgo agudo en uso normal. Los años de escolaridad presentaron una correlación altamente significativa $(\mathrm{p} \leq 00.1)$ con el empleo de plaguicidas de origen natural. Todos los entrevistados no utilizan equipo completo de protección personal y solo el $20 \%$ manifestó síntomas de intoxicación.

Palabras clave: conductas de uso, exposición laboral, plagas, fresas, sistemas de producción.

\begin{abstract}
The use and management of pesticides of different production systems were evaluated in Puebla and Tlaxcala States, Mexico. The methodology used was descriptive, qualitative and quantitative. Key informants were interviewed, who represented an average growing area of $2000 \mathrm{~m} 2$ and five production systems were characterized and were distinguished by the applied technology like irrigation system, hydroponic system and plastic padding soil as well as by growing under greenhouse condition and free exposure.
\end{abstract}

The results show that $100 \%$ of the products and day laborers use pesticides. The employees are frequently classified as less unlikely to present acute risk in normal use. The years of schooling showed a highly significant correlation ( $\mathrm{p} \leq 00.1$ ) with the usage of pesticides from natural origin. The interviewees do not use complete personal protection equipment and just the $20 \%$ manifested poisoning symptoms.

Key words: usage behavior, occupational exposure, pests, strawberries, production systems.

\footnotetext{
1. Doctor en Ciencias Naturales con especialidad en Biotecnología, Investigador, Facultad de Ciencias Biotecnoambientales, Universidad Popular Autónoma del Estado de Puebla, México; email: jesusantonio.salazar@upaep.mx

2. Ingeniero Agrónomo, Investigador, Facultad de Biotecnología, Universidad Popular Autónoma del Estado de Puebla, México; email: carloseduardo.somoza@upaep.edu.mx

3. Doctora en Ciencias con especialidad en Biotecnología, Directora, Facultad de Biotecnología, Universidad Popular Autónoma del Estado de Puebla, México; email: beatriz.perez@upaep.mx

4. Máster en Dirección Estratégica de Empresas, Decano, Facultad de Ingeniería y Arquitectura, Universidad Católica de El Salvador, El Salvador; email: mauricio.velasquez@catolica.edu.sv

5. Doctor en Ciencias, Representante técnico, Centro de Investigación en Alimentación y Desarrollo, México; email: gerardo.torres@ciad.mx 6. Doctor en Ciencias en Protección de Cultivos con especialidad en Control Biológico de Plagas, Profesor-Investigador asociado, Colegio de Posgraduados, Campus Puebla, México; email: arturohp@colpos.mx

7. Doctor en estrategias para el desarrollo agrícola, Consultor-Investigador, Universidad Popular Autónoma del Estado de Puebla, México; email: luisdaniel.ortega@upaep.mx * Autor para correspondencia
} 


\section{Introducción}

La fresa (Fragaria $x$ ananassa Duch.) es un cultivo de alto valor económico que se produce en campo abierto e invernadero, empleando sistemas de producción orgánicos y convencionales en los que se utilizan distintas tecnologías como macro y microtúneles, sustratos, acolchados y sistemas hidropónicos (Medellín et al., 2013; Rubio et al., 2014).

La fresa es un fruto no climatérico, altamente perecedero, susceptible a daños mecánicos, pérdidas de agua, deterioro fisiológico y microbiológico, lo que ocasiona la disminución en rendimiento y calidad comercial (Ruiz y Piedrahíta, 2012). Entre los principales problemas que los productores enfrentan durante la producción destacan las plagas y enfermedades, para su control se recurre a diferentes estrategias como el control biológico y extractos vegetales (Fan et al., 2009; Zong et al., 2010; Mekbib et al., 2011; Quezada, 2012); no obstante, esta estrategia carece de una respuesta clara, lo que se traduce en una falta de credibilidad de los agricultores (Milagrosa et al., 2010). De la misma forma se utilizan diferentes plaguicidas de síntesis química (Muiño et al., 2007; Aular y Casares, 2011) como bencimidazoles, dicarboximidas, anilinopirimidinas y carboxamidas (Russel, 2004), Pyraclostrobin más Epoxiconazole (Ayala et al., 2014). Sin embargo, en varios países se ha considerado a la fresa como uno de los productos agrícolas más contaminados por residuos de plaguicidas, y se ha indicado que su consumo puede constituir una importante ruta de exposición a una variedad de principios activos, incluyendo compuestos organoclorados (Fernandes et al., 2011; Vogt et al., 2012; Muñoz et al., 2014).

Un adecuado conocimiento del uso y manejo de los plaguicidas empleados en los sistemas de producción es un requisito básico, que constituye un elemento fundamental para orientar y estructurar estrategias que contribuyan a optimizar el uso de estos compuestos, sentando las bases para futuros estudios de evaluación de los efectos de estos productos en la salud humana y los ecosistemas. Además, para aportar información para las autoridades sanitarias y reguladoras que puedan intensificar esfuerzos en la capacitación y la actualización permanente del personal técnico, jornaleros y agricultores, así como fortalecer acciones de prevención y educación hacia la comunidad.

Por las razones expuestas, el objetivo del presente trabajo fue analizar, el uso y manejo de plaguicidas en diferentes sistemas de producción de fresa en los estados de Puebla y Tlaxcala, México.

\section{Materiales y Métodos}

La investigación se llevó a cabo en los municipios de Zacatlán: O $97^{\circ} 40$ - O 9800 / N $19^{\circ} 45$ 
- N 2000: 2040msnm; Huejotzingo: O $98^{\circ} 20$ O $98^{\circ} 40 / \mathrm{N} 19^{\circ} 00$ - N 19²15: 2274msnm; Cholula: $19^{\circ} 03^{\prime} \mathrm{N} 98^{\circ} 18^{\prime} \mathrm{O}: 2100 \mathrm{msnm}$ y Atlixco: O $98^{\circ} 20$ - O $98^{\circ} 40$ / N $18^{\circ} 45$ - N 1900: $1035 \mathrm{msnm}$ del estado de Puebla, y el municipio de Ixtenco: $19^{\circ} 15^{\prime} 00^{\prime \prime} \mathrm{N} 97^{\circ} 53^{\prime} 00^{\prime \prime O}$ : 2542msnm del estado de Tlaxcala (INEGI, 2014-2015).

La selección de los productores se basó en el inventario de invernaderos del estado de Puebla, (SAGARPA, 2008) y para su localización se empleó el método de muestreo "bola de nieve”, en donde se sitúan algunos individuos de la población y estos conducen a otros. Este método facilita la localización así como la creación de un vínculo de confianza que de otra forma habrían sido de difícil acceso (Crespo y Salamanca, 2007).

La información para el estudio se obtuvo empleando entrevistas semiestructuradas (investigación cualitativa); asimismo se implantó el uso del cuestionario aplicado (investigación cuantitativa) a cada productor por separado, el cual contenía preguntas cerradas y abiertas para facilitar la expresión de la opinión de los productores.

Se optó por basar la recolección de información sobre el último ciclo de cultivo que los productores hubiesen completado, esto para facilitar la adquisición de datos de recientes acontecimientos y situaciones que los mismos hubieran enfrentado. También, para facilitar las respuestas de los productores y tener da- tos de su actual realidad. Los datos obtenidos se analizaron con el paquete estadístico SPSS (Statical Package for the Social Sciences); para medir las variables cuantitativas se utilizaron medidas de tendencia central y de correlación de Spearman.

\section{Resultados y Discusión}

\section{Características de la población en estudio}

La población en estudio consistió en 33 personas, de las cuales seis fueron informantes clave (productores de fresa), quienes mencionaron emplear un promedio de 2.3 jornales por cada $1000 \mathrm{~m}^{2}$, cuatro horas de trabajo diarias. El 100\% de ellos pertenecía al sexo masculino, con un rango de edad de 26 a 60 años; y una desviación estándar de trece con promedio de 17.8 años de escolaridad; sabían leer y escribir. Todos los encuestados (100\%) refirieron utilizar y manipular plaguicidas y solo el 50\% realizaba actividades económicas distintas al invernadero, principalmente en el sector primario.

\section{Características de los sistemas de producción}

El 60\% de los encuestados produce fresa bajo condiciones de invernadero en una superficie promedio de $2000 \mathrm{~m}^{2}$; el resto de ellos lo realiza a libre exposición. Los sistemas de producción evaluados se clasificaron según sus componentes y tecnologías empleadas en el cultivo, (ver tabla 1). 
Tabla 1. Sistemas de producción de fresa encontrados en los estados de Puebla y Tlaxcala

\begin{tabular}{|c|c|c|c|c|c|c|}
\hline Sistema & $\begin{array}{c}\text { Hidroponía } \\
\text { con sustrato }\end{array}$ & Invernadero & $\begin{array}{c}\text { Sistema de } \\
\text { riego por } \\
\text { goteo }\end{array}$ & $\begin{array}{c}\text { Suelo con } \\
\text { acolchado } \\
\text { plástico }\end{array}$ & $\begin{array}{c}\text { Plaguici- } \\
\text { das y fer- } \\
\text { tilizantes } \\
\text { químicos }\end{array}$ & $\begin{array}{c}\text { Plaguici- } \\
\text { das y fer- } \\
\text { tilizantes } \\
\text { de origen } \\
\text { natural }\end{array}$ \\
\hline $\mathrm{A}$ & & & $\mathrm{X}$ & $\mathrm{X}$ & $\mathrm{X}$ & \\
\hline $\mathrm{B}$ & $\mathrm{X}$ & & $\mathrm{X}$ & & $\mathrm{X}$ & \\
\hline $\mathrm{C}$ & & $\mathrm{X}$ & $\mathrm{X}$ & $\mathrm{X}$ & $\mathrm{X}$ & \\
\hline $\mathrm{D}$ & $\mathrm{X}$ & $\mathrm{X}$ & $\mathrm{X}$ & & $\mathrm{X}$ & \\
\hline $\mathrm{E}$ & & $\mathrm{X}$ & $\mathrm{X}$ & $\mathrm{X}$ & & $\mathrm{X}$ \\
\hline
\end{tabular}

Fuente: Elaboración propia con información de campo.

Plaguicidas utilizados durante el ciclo agrícola de producción 2016

Los plaguicidas empleados con mayor frecuencia son los clasificados como poco probable de presentar riesgo agudo en uso normal, seguidos de los moderadamente peligrosos, según la clasificación de peligrosidad de WHO (2009) (Tabla 2). El tipo de plaguicida más utilizado es el insecticida, así como extractos vegetales y microorganismos, principalmente para el control y eliminación de Bemisia tabaci (mosquita blanca), debido a que el 100\% de los informantes mencionaron que esta es la principal plaga.

Tabla 2. Plaguicidas utilizados en los diferentes sistemas de producción de fresa

\begin{tabular}{|l|c|c|}
\hline \multicolumn{3}{|c|}{ Sistema de cultivo en suelo bajo condiciones de invernadero } \\
\hline \multicolumn{1}{|c|}{ Ingrediente activo } & Tipo & Clasificación de peligrosidad \\
\hline Argemonina, Berberina Ricinina, A-Terthienyl & I & IV \\
\hline Cipermetrina & I & III \\
\hline Hidróxido cúprico & F & II \\
\hline Agrimec, Abamectina & I & III \\
\hline Baytroid, Cyflutrin & I & III \\
\hline Ciantraniliprol & I & III \\
\hline Clorotalonil, Tetracloroisoftalonitrilo & F & II \\
\hline Cymoxanil+hidroxico cúprico & F & II \\
\hline Dimetomorf & F & II \\
\hline
\end{tabular}


Sistema de cultivo hidropónico en invernadero

\begin{tabular}{|c|c|c|}
\hline Ingrediente activo & Tipo & Clasificación de peligrosidad \\
\hline $\begin{array}{l}\text { Oxamil: S-metil N'N'-dimetil-N- } \\
\text { (metilcarbamoiloxi)- 1-tio-oxamimidato }\end{array}$ & I & IV \\
\hline $\begin{array}{l}\text { Imidacloprid: (E)-1-(6-cloro-3-piridilmetil) -N- } \\
\text { nitroimidazolidin-2-ilidenamina }\end{array}$ & I & IV \\
\hline $\begin{array}{l}\text { Alga marina eklonia máxima, Ácido indol-3-butíri- } \\
\text { co (4-(1 H-indol-3-yl) butyric acid) }\end{array}$ & $\mathrm{Fi}$ & \\
\hline $\begin{array}{l}\text { Metalaxil: N-(metoxiacetil)-N-(2,6-xilil)-DL-alani- } \\
\text { nato + Mancozeb: Manganeso ethylenebis (dithio- } \\
\text { carbamate) (polymeric) complejo con sal de zinc }\end{array}$ & $\mathrm{F}$ & IV \\
\hline Carbendazim: Metilbenzimidazol-2-il carbamato & $\mathrm{F}$ & III \\
\hline Metilen bistiocianato +2 - tiociantametiltio & $\mathrm{B}$ & II \\
\hline Polisacaridos y MOs & $\mathrm{Fi}$ & I \\
\hline Extractos halogenados & & I \\
\hline Quillaja & & I \\
\hline Paecilomyces lilacinus & ME & I \\
\hline \multicolumn{3}{|c|}{ Sistema de cultivo hidropónico a campo abierto } \\
\hline Ingrediente activo & Tipo & Clasificación de peligrosidad \\
\hline Extracto de ajo & $\mathrm{PO}$ & $\mathrm{I}$ \\
\hline Metarhizuim & ME & I \\
\hline Bacillus subtilis QST 713 & $\mathrm{ME}$ & I \\
\hline Dióxido de cloro & $\mathrm{D}$ & II \\
\hline Extracto alcohólico de mimosa + Quercus & & $\mathrm{I}$ \\
\hline Extracto de Larrea tridentata $L$. & $\mathrm{PO}$ & $\mathrm{I}$ \\
\hline Flonicamid & I & III \\
\hline \multicolumn{3}{|c|}{ Suelo con acolchado plástico campo abierto } \\
\hline Ingrediente activo & Tipo & Clasificación de peligrosidad \\
\hline Mancozeb & $\mathrm{F}$ & IV \\
\hline Rhizobac combi & $\mathrm{BiF}$ & I \\
\hline Insecticidas orgánicos & $\mathrm{PO}$ & $\mathrm{I}$ \\
\hline \multicolumn{3}{|l|}{ Ácido Fosfórico } \\
\hline Lixiviado de Lombriz & $\mathrm{PO}$ & I \\
\hline \multicolumn{3}{|c|}{ Sistema de producción Orgánica en Suelo } \\
\hline Ingrediente activo & Tipo & Clasificación de peligrosidad \\
\hline Hidróxido de Calcio & & I \\
\hline Metarhizium anisopliae & ME & I \\
\hline Extractos Vegetales & $\mathrm{PO}$ & I \\
\hline
\end{tabular}




\begin{tabular}{|l|c|c|}
\hline \multicolumn{3}{|c|}{ Sistema de producción Orgánica en Suelo } \\
\hline \multicolumn{1}{|c|}{ Ingrediente activo } & Tipo & Clasificación de peligrosidad \\
\hline Trichoderma spp & ME & I \\
\hline Micorrizas & PO & I \\
\hline Humus de lombriz & PO & I \\
\hline Trichoderma harzarium, Cepa T-22. & ME & I \\
\hline Harina de pescado & PO & I \\
\hline Amino-ácidos & Fi & I \\
\hline Ácidos húmicos y fúlvicos & Fi & I \\
\hline Beauveria bassiana & ME & I \\
\hline Larvicidas hechos a base de Bacillus Thuringensis & ME & I \\
\hline Bacillus subtilis y linqueniforme & ME & I \\
\hline Lactobacilus & PO & I \\
\hline
\end{tabular}

Nota: Según WHO (2009), la clasificación de peligrosidad: $\mathrm{Ia}=$ extremadamente peligroso, $\mathrm{Ib}=$ altamente peligroso, $\mathrm{II}=$ moderadamente peligroso, $\mathrm{III}=$ ligeramente peligroso, $\mathrm{U}=$ poco probable que presente riesgo agudo en uso normal. Mecanismo de acción: $\mathrm{C}=$ contacto, $\mathrm{S}=$ sistémico, $\mathrm{I}=$ ingestión, $\mathrm{H}=$ inhalación.

Fuente: Elaboración propia con información de campo.

El 100\% de los encuestados utiliza plaguicidas para el control de insectos, siendo el grupo químico más aplicado el de los carbamatos y ditiocarbamatos, seguido de los compuestos de cobre, piretroides, entre otros. Asimismo, utilizan extractos vegetales y de diferentes microorganismos, principalmente hongos entomopatógenos, (ver Tabla 3).

Los plaguicidas de mayor grado de peligrosidad son empleados en sistema D; asimismo, el productor refirió como grado máximo de estudios a nivel de secundaria (9 años de escolaridad); mientras que los restantes sistemas de producción utilizan plaguicidas, a excepción del E. Por otro lado, la edad es un factor a considerar en el uso de plaguicidas y la elección del sistema de producción, ya que existe una correlación altamente significativa entre el uso de plaguicidas de origen natural y la edad de los productores (Figura 1) (ver Tabla 4). 
Tabla 3. Ingrediente activo, fórmula y grupo químico de principales plaguicidas empleados en sistemas de producción de fresa

\begin{tabular}{|c|c|c|}
\hline Ingrediente activo & Fórmula química & Grupo químico \\
\hline \multicolumn{3}{|c|}{ Sistema de cultivo en suelo bajo condiciones de invernadero } \\
\hline $\begin{array}{l}\text { Argemonina, Berberina Rici- } \\
\text { nina, A-Terthienyl }\end{array}$ & $\mathrm{N} / \mathrm{A}$ & Insecticida botánico \\
\hline Cipermetrina & $\mathrm{C}_{22} \mathrm{H}_{19} \mathrm{C}_{12} \mathrm{NO}_{3}$ & Piretroide \\
\hline Hidróxido cúprico & $\mathrm{Cu}(\mathrm{OH})_{2}$ & Cúpricos \\
\hline Agrimec, Abamectina & $\begin{array}{l}\mathrm{C}_{48} \mathrm{H}_{72} \mathrm{O}_{14}(\mathrm{~B} 1 \mathrm{a})+\mathrm{C}_{47} \mathrm{H}_{70} \mathrm{O}_{14} \\
(\mathrm{~B} 1 \mathrm{~b})\end{array}$ & $\begin{array}{l}\text { Avermectinas, lactona ma- } \\
\text { crocíclica }\end{array}$ \\
\hline Baytroid, Cyflutrin & $\mathrm{C}_{22} \mathrm{H}_{18} \mathrm{C}_{12} \mathrm{FNO}_{3}$ & Piretroide \\
\hline Ciantraniliprol & $\mathrm{C}_{19} \mathrm{H}_{14} \mathrm{BrC}_{1} \mathrm{~N}_{6} \mathrm{O}_{2}$ & Diamidas Antranílicas \\
\hline $\begin{array}{l}\text { Clorotalonil, Tetracloroisof- } \\
\text { talonitrilo }\end{array}$ & $\mathrm{C}_{8} \mathrm{C}_{14} \mathrm{~N}_{2}$ & Cloronitrilos \\
\hline Cymoxanil+hidroxico cúprico & $\mathrm{C}_{7} \mathrm{H}_{10} \mathrm{~N}_{4} \mathrm{O}_{3}+\mathrm{CU}(\mathrm{OH})_{2}$ & $\begin{array}{l}\text { Cianoacetamida oxima + Cú- } \\
\text { prico }\end{array}$ \\
\hline Dimetomorf & $\mathrm{C}_{21} \mathrm{H}_{22} \mathrm{C}_{1} \mathrm{NO}_{4}$ & Morfolina, Clorado. \\
\hline \multicolumn{3}{|c|}{ Sistema de cultivo hidropónico en invernadero } \\
\hline $\begin{array}{l}\text { Oxamil: S-metil N'N'N-dime- } \\
\text { til-N-(metilcarbamoiloxi)- } \\
\text { 1-tio-oxamimidato }\end{array}$ & $\mathrm{C}_{7} \mathrm{H}_{3} \mathrm{~N}_{3} \mathrm{O}_{3} \mathrm{~S}$ & Carbamato \\
\hline $\begin{array}{l}\text { Imidacloprid: (E)-1-(6-cloro- } \\
\text { 3-piridilmetil) -N-nitroimi- } \\
\text { dazolidin-2-ilidenamina }\end{array}$ & $\mathrm{C}_{9} \mathrm{H}_{10} \mathrm{C}_{1} \mathrm{~N}_{5} \mathrm{O}_{2}$ & Neonicotinoides \\
\hline $\begin{array}{l}\text { Alga marina eklonia máxima, } \\
\text { Ácido indol-3-butírico (4-(1 } \\
\text { H-indol-3-yl) butyric acid) }\end{array}$ & $\mathrm{N} / \mathrm{A}$ & Producto orgánico \\
\hline $\begin{array}{l}\text { Carbendazim: Metilbenzimi- } \\
\text { dazol-2-il carbamato }\end{array}$ & $\mathrm{C}_{9} \mathrm{H}_{9} \mathrm{~N}_{3} \mathrm{O}_{2}$ & Benzimidazole \\
\hline $\begin{array}{l}\text { Metilen bistiocianato }+2- \\
\text { tiociantametiltio }\end{array}$ & $\mathrm{CH}_{2}(\mathrm{SCN})_{2}$ & \\
\hline Polisacaridos y MOs & $\mathrm{N} / \mathrm{A}$ & Producto orgánico \\
\hline Extractos halogenados & $\mathrm{N} / \mathrm{A}$ & Átomos de halógeno \\
\hline Quillaja & N/A & Saponinas \\
\hline Paecilomyces lilacinus & N/A & Hongo entomopatógeno \\
\hline
\end{tabular}




\begin{tabular}{|c|c|c|}
\hline Ingrediente activo & Fórmula química & Grupo químico \\
\hline \multicolumn{3}{|c|}{ Sistema de cultivo hidropónico a campo abierto } \\
\hline Extracto de ajo & N/A & Producto orgánico \\
\hline Metarhizuim & N/A & Hongo entomopatógeno \\
\hline Bacillus subtilis QST 713 & N/A & Bacteria entomopatógena \\
\hline Dióxido de cloro & $\mathrm{C}_{1} \mathrm{O}_{2}$ & \\
\hline $\begin{array}{l}\text { Extracto alcohólico de mimo- } \\
\text { sa + Quercus }\end{array}$ & N/A & Fungicida bioagrícola \\
\hline Extracto de Larrea tridentata $L$. & N/A & Producto orgánico \\
\hline Flonicamid & $\mathrm{C}_{9} \mathrm{H}_{6} \mathrm{~F}_{3} \mathrm{~N}_{3} \mathrm{O}$ & Piridinocarboxamidas \\
\hline \multicolumn{3}{|c|}{ Suelo con acolchado plástico campo abierto } \\
\hline Mancozeb & C8H12MnN4S8Zn & Ditiocarbamato \\
\hline Rhizobac combi & N/A & Producto orgánico \\
\hline Insecticidas orgánicos & N/A & Producto orgánico \\
\hline Ácido Fosfórico & $\mathrm{H}_{3} \mathrm{PO}_{4}$ & Oxácidos \\
\hline Lixiviado de Lombriz & N/A & Producto orgánico \\
\hline \multicolumn{3}{|c|}{ Sistema de producción Orgánica en Suelo } \\
\hline Hidróxido de Calcio & $\mathrm{Ca}(\mathrm{OH})_{2}$ & \\
\hline Metarhizium anisopliae & N/A & Hongo entomopatógeno \\
\hline Extractos Vegetales & N/A & Producto orgánico \\
\hline Trichoderma spp & N/A & Hongo fungistático \\
\hline Micorrizas & N/A & Producto orgánico \\
\hline Humus de lombriz & N/A & Producto orgánico \\
\hline $\begin{array}{l}\text { Trichoderma harzarium, } \\
\text { Cepa T-22. }\end{array}$ & N/A & Hongo fungistático \\
\hline Harina de pescado & N/A & Producto orgánico \\
\hline Amino-ácidos & N/A & N/A \\
\hline Ácidos húmicos y fúlvicos & N/A & Producto orgánico \\
\hline Beauveria bassiana & N/A & Hongo entomopatógeno \\
\hline $\begin{array}{l}\text { Larvicidas hechos a base de } \\
\text { Bacillus Thuringensis }\end{array}$ & $\mathrm{N} / \mathrm{A}$ & Bacteria entomopatógena \\
\hline Bacillus subtilis y linqueniforme & $\mathrm{N} / \mathrm{A}$ & Bacteria entomopatógena \\
\hline Lactobacilus & N/A & Producto orgánico \\
\hline
\end{tabular}


Tabla 4. Escolaridad en años y porcentaje de plaguicidas utilizados en los sistemas de producción

\begin{tabular}{|c|c|c|c|}
\hline \multirow{2}{*}{ Sistema de producción } & \multirow{2}{*}{ Escolaridad en años } & \multicolumn{2}{|c|}{ Porcentaje de plaguicidas utilizados } \\
\cline { 3 - 4 } & & Orgánicos & Químico \\
\hline Suelo bajo invernadero & 16 & 30 & 70 \\
\hline Hidropónico en invernadero & 9 & 20 & 80 \\
\hline hidropónico a campo abierto & 20 & 50 & 50 \\
\hline Suelo acolchado a campo abierto & 20 & 60 & 40 \\
\hline Orgánico en Suelo & 24 & 100 & 0 \\
\hline
\end{tabular}

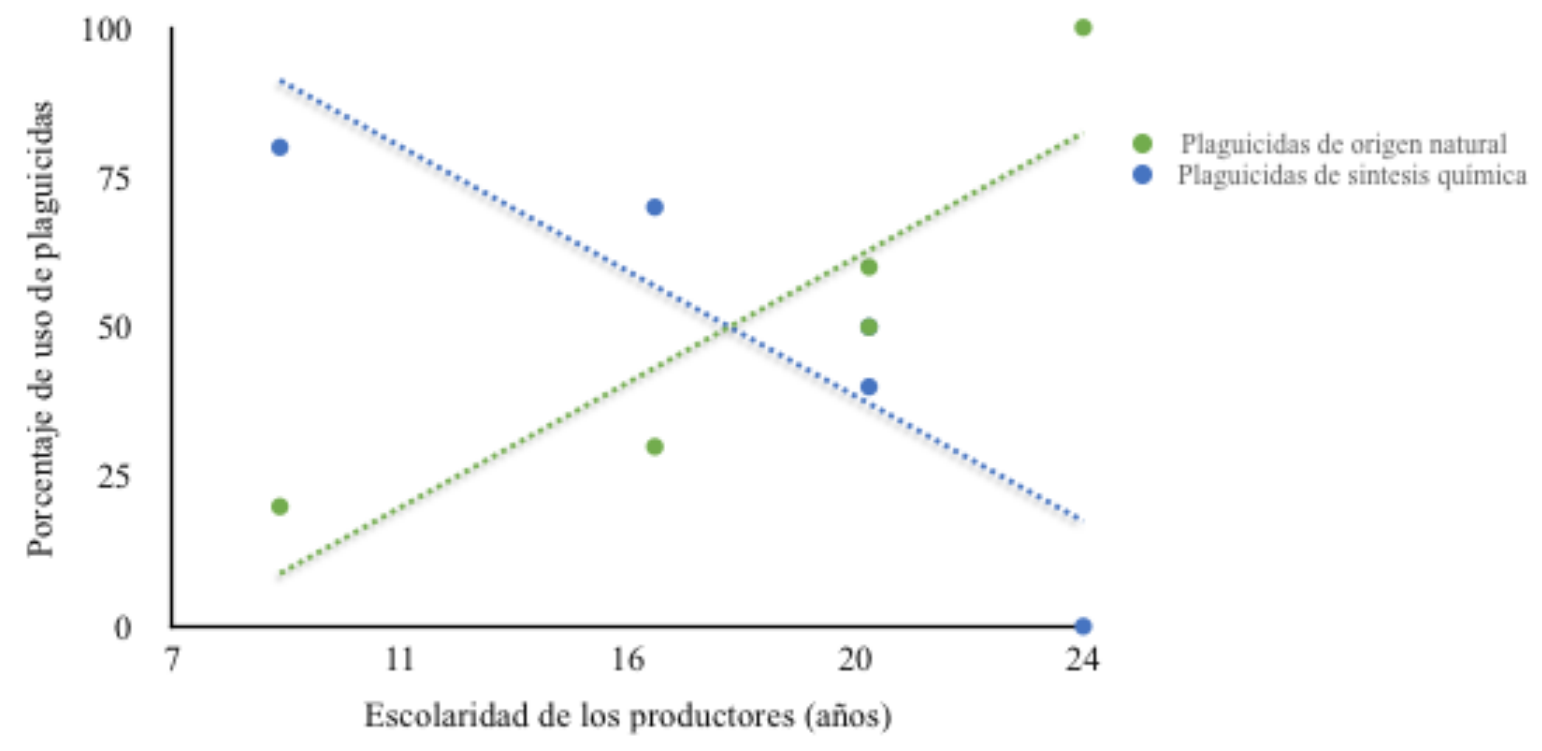

\begin{tabular}{|c|c|c|}
\hline & $\begin{array}{l}\text { Plaguicidas de } \\
\text { origen natural }\end{array}$ & $\begin{array}{l}\text { Plaguicidas de } \\
\text { sintesis quimica }\end{array}$ \\
\hline Escolaridad & $894^{\circ}$ &,$- 894^{*}$ \\
\hline Orgánicos & 1 & $-1,000^{* *}$ \\
\hline
\end{tabular}

Figura 1. Correlación entre el uso de plaguicidas orgánicos y sintéticos con los años de escolaridad $\mathrm{p}=0.050 .01$. 


\section{Asistencia técnica}

El 100\% de los encuestados refirió asistencia técnica en la producción del cultivo; por otro lado, los jornaleros que laboran en sus unidades de producción destinados a la acción de aplicación de plaguicidas, no utiliza el equipo de protección personal completo, el cual consiste en: mascarilla, anteojos, guantes, ropa adecuada y botas (Tabla 4), aumentando el riesgo y las probabilidades de que sufran una intoxicación e inclusive la pérdida de la vida.

El 80\% de los jornaleros ingiere líquidos o alimentos en horas laborales y en el sitio del cultivo; a su vez manifestaron no presentar síntomas de intoxicación como vómito, mareos, ronchas y ardor, únicamente 20\% manifestó haber presentado cefalea posterior a la aplicación de plaguicidas, aunque no de manera constante, (ver Tabla 5).
El 100\% de los encuestados refirió que los jornaleros que laboran en sus unidades de producción no utilizan ropa exclusiva para las aplicaciones de plaguicidas, la cual consiste en un pantalón, camisa o playera de tela común y zapatos. La ropa que utiliza es empleada en varias y constantes ocasiones antes de ser lavada. El $80 \%$ de los encuestados no se cambia de ropa posterior a la aplicación; y cuando ésta se lava no se hace por separado de otras prendas personales e incluso familiares. Además, no se lavan los equipos de protección como guantes, cubre bocas, botas, entre otros. Asimismo, refirieron no reingresar al invernadero o área de cultivo antes de tres horas para continuar con labores agronómicas.

Por otra parte, el 100\% de los jornaleros ingresa a las labores antes de las doce horas posteriores a la aplicación; también entran otras personas ajenas para realizar activida-

Tabla 5. Indicadores de uso y manejo de plaguicidas

\begin{tabular}{|l|c|}
\hline \multicolumn{1}{|c|}{ Variable } & Porcentaje \\
\hline Lee la recomendación del uso del plaguicidas & 80 \\
\hline Emplea equipo de protección completa & 0 \\
\hline Utiliza ropa exclusiva para la aplicación de plaguicidas & 0 \\
\hline Acostumbra cambiar de ropa después de la aplicación & 0 \\
\hline Baño posterior al uso de plaguicidas & 20 \\
\hline Después de la aplicación lava las prendas de protección & 80 \\
\hline Lava por separado la ropa que utiliza en la aplicación & 0 \\
\hline Ingresa al área de cultivo antes de 12 horas posteriores a la aplicación de plaguicidas & 100 \\
\hline Ingresa al área de cultivo antes de tres horas posteriores a la aplicación de plaguicidas & 100 \\
\hline Intoxicación con plaguicidas & 20 \\
\hline Síntomas de intoxicación posterior a utilizar plaguicidas & 50 \\
\hline Consume el producto que cultiva & 100 \\
\hline
\end{tabular}


des de la producción de fresa, o entran personas que no están involucradas en las labores de cultivo.

\section{Almacenamiento de plaguicidas}

En el almacenamiento de los plaguicidas, el $100 \%$ de los productores lo hace en instalaciones ajenas al área del sistema de cultivo: un $20 \%$ lo hace en su casa y el restante en bodegas, aunque esta última no sea única y especial para los plaguicidas. El 100\% de los productores mencionó no tener sobrantes de plaguicidas, pues utilizan las dosis exactas para cubrir su cultivo; sin embargo, cuando no utilizan el total de producto, este es guardado en su envase original, botellas de PET o bolsas de plástico. Los envases vacíos son recolectados por el servicio de limpia sin el tratamiento previo y adecuado por el productor.

La producción de fresa es una actividad reciente en la zona de estudio. Los productores implementan distintas tecnologías como invernaderos, sistemas de riego y producción; resultados similares a éstos fueron obtenidos por Ortega et al. (2014), no obstante, la actividad se desarrolló con el uso intensivo de plaguicidas. Estas observaciones también coincidieron con las reportadas en otros estudios (Dara et al., 2016; Surendra et al., 2016; Michał et al., 2015).

En lo que se refiere a los resultados obtenidos, en el cultivo de fresa con los diferentes sistemas de producción, existe una similitud en los distintos grupos químicos con lo reportado por Pérez et al. (2015). Esto muestra que la alta incidencia de plagas y en específico de la mosquita blanca en las fresas son combatidas por los productores por una amplia diversidad de agroquímicos, incrementando el riesgo a la exposición y posiblemente de dejar residuos.

En este sentido, en este trabajo observamos tres aspectos relevantes a considerar: la tecnología empleada para la producción, el manejo agronómico del cultivo y las características agroclimáticas de la zona de estudio, que influyen en la selección de los agroquímicos. Cabe destacar, sin embargo, que los productores emplean extractos vegetales y control biológico en todos los sistemas a pesar de que la agricultura orgánica tiene una historia de ser contenciosa, y es considerada por algunos como un enfoque ineficiente para la producción de alimentos, tal y como lo mencionan Reganold et al. (2015).

Sin embargo, estudios recientes muestran que la producción de alimentos orgánicos y bebidas es un segmento de mercado en rápido crecimiento dentro de la industria alimentaria mundial, a diferencia de los rendimientos en la producción de orgánica, que es más baja en comparación con la agricultura convencional, con las ventajas de ser más rentables y respetuoso con el medio ambiente. En este 
sentido, el cliente recibe alimentos iguales o más nutritivos que contienen menos (o ningún) plaguicida.

Los alimentos orgánicos permiten el uso de plaguicidas de origen natural y control biológico los cuales, aunados al orden y limpieza, logran controlar los umbrales mínimos de daño de plagas en el área de cultivo (Fan et al., 2009; Zong et al., 2010; Mekbib et al., 2011; Quezada, 2012). En lo que respecta a México, Pérez Olvera et al. (2011) han reportado que de los controladores de plagas usados, el $6.2 \%$ corresponde a los de tipo biológico empleando especies como Bacillus thuringiensis, Trichogramma sp., Isaria fumosorosea y Beauveria bassiana.

En los jornaleros, el uso de plaguicidas puede influir en actividad genotóxica, lo cual constituye un factor de riesgo para la población expuesta, ya que se sabe de la estrecha relación que existe entre daño genotóxico y aparición de enfermedades como el cáncer (Pabuena et al, 2016). En mayor o menor grado, la población humana está inevitablemente expuesta a pesticidas, los cuales contribuyen a la contaminación ambiental por medio de productos degradados en aire, suelo, agua y alimentos; este contacto a largo plazo puede inducir daño en las poblaciones, perturbando órganos, tejidos, sistemas entre otros (Collota et al., 2013).
Las diferencias en los sistemas de producción generan condiciones bióticas y abióticas distintas en el área de cultivo. En los sistemas de invernaderos, las condiciones que se generan en el interior - principalmente altas temperaturas y humedad relativa - potencian la toxicidad de los productos plaguicidas (Palomar, 1993). Las condiciones de temperatura incomodan la utilización del equipo de protección y las medidas preventivas recomendadas para el uso de los plaguicidas, lo que aumenta la exposición a dichas sustancias como lo menciona Ortega et al. (2014). Sin embargo, los jornaleros a libre exposición - donde las condiciones ambientales son diferentes al interior del invernadero - no utilizan el equipo de protección completo, exponiéndose a moléculas tóxicas por las vías respiratorias, dérmicas o digestivas, como lo mencionan Gómez, Díaz, Meneses, Villalobos y De León (2000).

Al evaluar a un grupo de trabajadoras en invernadero en la floricultura mexicana expuestas a plaguicidas, de la misma forma Ortega et al., (2017), mencionan el daño genotóxico en jornaleros expuestos a plaguicidas en invernaderos. Sammons et al. (2005) indican que la exposición está condicionada por la variabilidad de tiempo de exposición y éste, a su vez, por la superficie del área de cultivo. Para nuestro estudio, un jornal está expuesto laboralmente un promedio de cuatro horas diarias, con diferentes actividades 
propias del sistema de cultivo, resultado que difiere de los tiempos de Requena (2009), quien señala que el trabajo por hectárea de invernadero en España puede oscilar entre doce y catorce horas; y con una variabilidad en función de que el trabajador sea fumigador habitual o esporádico.

La disposición, tratamiento y recolección final de los residuos sólidos y biológicos en la zona de estudio coinciden con las acciones mencionadas por Martínez et al. (2009); Escobar et al. (2011); y Ortega et al. (2014), en los estados de Sinaloa, Chiapas y Puebla respectivamente. Es importante enfatizar que los recipientes al ser entregados al servicio de limpia no garantiza el tratamiento especial y los plaguicidas presentes en los plásticos permanecen sin sufrir ningún proceso de degradación, lo que debe tomarse en cuenta a la hora de manipular este material contaminado o disponer los residuos en vertederos, mediante incineración o reciclaje.

\section{Conclusiones}

El desarrollo de las prácticas agrícolas por parte de los productores de fresa se realizó en cinco sistemas de producción diferenciados por las tecnologías implementadas, que se caracterizan por un uso intensivo y por una gran variedad de plaguicidas. Los años de estudio fueron una variable que influye en el uso de plaguicidas de origen natural y control biológico.

El uso de plaguicidas no sólo afecta al productor o jornaleros que laboran en el área de cultivo y que sufre la mayor exposición, sino también al ambiente y a la población en general. Esto hace necesario instar, desarrollar, implementar y fomentar estrategias enfocadas a la capacitación y concientización, que conlleven a su mejor uso y manejo, así como a nuevas técnicas agrícolas para racionalizar la necesidad de aplicación de los agroquímicos.

\section{Referencias}

Aular, J. y Casares, M. (2011). Consideraciones sobre la producción de frutas en Venezuela. Rev. Bras. Frutic. 33 (nº espec.), pp.187-198

Ayala, M.; Almanza; Merchan, J.; Serrano, C. (2014). Efecto de Pyraclostrobin+Epoxiconazole en la producción de fresa (Fragaria sp.). Ciencia y agricultura, pp. 35-45

Collotta, M.; Bertazzi, P. A. y Bollati, V. (2013). Epigenetics and pesticides. Toxicology, pp 1-7.

Crespo, C. y Salamanca, C. (2007). El muestreo en la investigación cualitativa. Revista Nure Investigación, 27(2), pp.1-4. Kosmalaa. Recuperado de http://dx.doi. org/10.1016/j.nfs.2015.09.001 
Dara, S. K.; Sandoval-Solis, S. y Peck, D. (2016). Improving Strawberry Irrigation with MicroSprinklers and Their Impact on Pest Management. Agricultural Sciences, 7(12), p. 859

Escobar, D.; Caballero, A. y Rendón, V. (2011). Prácticas de utilización para plaguicidas en la localidad Nueva Libertad, La Concordia, Chiapas. Revista Mexicana de Ciencias Agrícolas, 1(4), pp. 19-30

Fan, Y.; Xu, Y.; Wang, D.; Zhang, L.; Sun, J. y Sun, L. 2009. Effect of alginatecoating combined with yeast antagonist on strawberry (Fragariaxananassa) preservation quality. Postharvest Biol. 58, pp. 84-90

Fernandes, V.; Domingues, N.; Mateus y Deluere-Matos C. (2011). Organochlorine pesticide residues in strawberries from integrated pest management and organic farmin. $J$. Agric. Food Chem. 59(14), pp.7582-7591.

Gómez, S., Díaz, S., Meneses, P., Villalobos, R. y De León, R., (2000). Cytogenetic biomonitoring in a Mexican floriculture workers group exposed to pesticides. Mutation Research, 466(1), pp.117-124. Recuperado de http://www.sciencedirect.com/science/article/pii/ S092552140900060X

Martínez-Valenzuela, C., Gómez-Arroyo, S., Villalobos-Pietrini, R., Waliszewski, S., CalderónSegura, M.E., Félix-Gastélum y R., Álvarez-Torres, A. (2009). Genotoxic biomonitoring of agricultural workers exposed to pesticides in the north of Sinaloa State, Mexico. Environ. Int. 35(8), pp. 1155-1159

Medellín C., L. A. (2013). Evaluación de Materiales para el Acolchado de la Fresa Cultivada Bajo Invernadero. Revista Facultad de Ciencias Básicas, [S.1.], v. 9, n. 1, pp. 8-19. ISSN 1900-4699. Recuperado de https://revistas.unimilitar.edu.co/index.php/rfcb/article/ view/352

Mekbib, S.; Regnier, T. y Korsten, L. (2011). Efficacy and mode of action of yeast 311 antagonists for control of Penicillium digitatum in oranges. Plant Pathol. 36: 312, pp. 233-240.

México, Secretaría de Agricultura Ganadería Desarrollo Rural Pesca y Alimentación (2008). Inventario de invernaderos del estado de Puebla. Recuperado de http://www.oeidruspuebla.gob.mx/RID. pdf 06/02/2010 
Michał, S.; Artur, M.; Piotr S.; Katarzyna Z.; Elżbieta K. y Monika K. (2015). Pesticide residue levels in strawberry processing by-products that are rich in ellagitannins and an assessment of their dietary risk to consumers. Recuperado de http://doi.org/10.1016/j. nfs.2015.09.001

Milagrosa S., F. D.; Cara, M. d.; Camacho, F. y Tello, J. C. (2010). El control biológico de plagas y enfermedades. Un encuadre crítico. Cuadernos de 321 estudios agroalimentarios, pp. $61-72$

Muiño, E.; Botta, E.; Pérez, A.; Ballester, D.; Moreno, F.; Rodríguez, E. y Fernández, R. (2007). Sistemas de manejo integrado de plagas como alternativa al uso del bromuro de metilo en la producción de cultivos protegidos, flores y ornamentales. Boletín Fitosanitario, 12(1), pp. 1-71

Muñoz-Quezada, M.; Lucero, B.; Iglesias, V. y Muñoz M. (2014). Vías de exposición a plaguicidas en escolares de la Provincia de Talca, Chile. Gac. Sanit. 28(3), pp. 90-195

Ortega M., L. D.; Martínez V., C.; Waliszewski, S.; Ocampo M., J.; Huichapan M., J.; El Kassis, E.; Soto R., G. y Pérez A., B. (2017). Nivel tecnológico de invernadero y riesgo para la salud de los jornaleros

Pabuena, D.; Ortiz, I.; López, J.; Orozco, L.; Parra, A. Q.; Pardo, E. y Meléndez, I. (2016). Actividad genotóxica inducida por extracto de fresa fumigada con pesticidas en pamplona, norte de santander, Colombia. Universidad Ciencia y Tecnología, 19(76)

Palomar, O. (1993). Los invernaderos y el medio ambiente (1ª ed). Almería, España: Cantón

Pérez, R.; Salas, J. y Amaro, R. (2015). Comparación de dos métodos de extracción para la determinación de pesticidas organoclorados y organofosforados en fresas. CIENCIA 23 (3), pp. 158-167, Maracaibo, Venezuela

Pérez-Olvera, M. A.; Navarro-Garza, H. y Miranda-Cruz, E. (2011). Use of pesticides for vegetable crops in Mexico. En: Pesticides in the Modern World-Pesticides Use and Management (M. Stoytcheva, Ed.). In Tech, Rijeka, Croacia, pp. 97-118 
Quezada, M. Q. y Patricia, A. (2012). Evaluación del comportamiento de fungicidas microbiológicos en la prevención de Botritis en el cultivo de fresa (Fragaria 344 Vesca). Gestión de la Producción de Flores y Frutas Andinas para 345 Exportación. (Tesis maestría): universidad técnica de Ambato

Reganold, J. P. y Wachter, J. M. (2015). Organic agriculture in the twenty-first century Nature plant. Article number: 15221 | DOI: 10.1038/nplants.2015.221

Requena, M. (2009). Estudio andaluz de prevalencia de diversas patologías en áreas con distinto nivel de utilización de plaguicidas (Tesis de Doctorado). Universidad de Granada: España.

Rubio, A.; Alfonso, A. M.; Grijalba, C. M. y Pérez, M. M. (2014). Determinación de los costos de producción de la fresa cultivada a campo abierto y bajo macrotúnel. DOI: https://doi. org/10.17584/rcch.2014v8i1.2801

Ruiz, R., Piedrahita, W. (2012). Manual para el cultivo de frutales en el trópico. 352 Produmedios, editor. Bogota

Russel, P. (2004). Sensitivity baselines in fungicide resistance research and 353 management. Brussels Gog Magog House.

Sammons, P. J.; Furukawa, T. y Bulgin, A. (2005). Autonomous Pesticide Spraying Robot for use in a Greenhouse. Paper presented in Australasian Congress Robotics and Automation Sydney, Australia.

Surendra K.; Dara, Samuel Sandoval-Solis, David Peck (2016). Improving Strawberry Irrigation with Micro-Sprinklers and Their Impact on Pest Management. Agricultural Sciences $07: 12$, pp. $859-868$

Vogt, R.; Bennett, D.; Cassady, J.; Frost, B.; Ritz y I. Hertz-Picciotto. (2012). Cancer and noncancer health effects from food contaminant exposures for children and adults in $\mathrm{Ca}$ lifornia: a risk assessment. Env. Health 11(83), pp. 2-14.

World Health Organization (2009). The WHO recommended classification of pesticides by hazard and guidelines to classification, Stuttgart, Alemannia: World Health Organization.

Zong, Y.; Liu, J.; Li, B.; Qin, G.; Tian, S. (2010). Effects of yeast antagonists in combination with hot water treatment on postharvest diseases of tomato fruit. Biol. Control. 54, pp. 316-321. 